\title{
Erratum to: Fibrinogen production is enhanced in an in-vitro model of non-alcoholic fatty liver disease: an isolated risk factor for cardiovascular events?
}

Emily N. W. Yeung ${ }^{1 * \dagger}$, Philipp Treskes ${ }^{1 \dagger}$, Sarah F. Martin², Jonathan R. Manning ${ }^{3}$, Donald R. Dunbar ${ }^{3}$, Sophie M. Rogers ${ }^{2}$, Thierry Le Bihan², K. Ann Lockman', Steven D. Morley ${ }^{1}$, Peter C. Hayes ${ }^{1}$, Leonard J. Nelson ${ }^{1}$ and John N. Plevris ${ }^{1}$

\section{Erratum}

After publication of the original article [1], it came to the authors' attention that sources of funding for the study presented were inadvertently omitted. The Acknowledgements section should therefore have read as follows:

'This work was supported by the Biotechnology and Biological Sciences Research Council [grant number BB/ L023687/1]. JRM \& DRD were supported by the British Heart Foundation Centre of Research Excellence award RE/13/3/30183. The decision to submit the article for publication was not influenced by the funding bodies.'

\footnotetext{
Author details

${ }^{1}$ Hepatology Laboratory, Division of Health Sciences, University of Edinburgh,

Chancellor's Building, 49 Little France Crescent, Edinburgh EH16 4SB, UK.

${ }^{2}$ Kinetic Parameter Facility, SynthSys, Centre for Synthetic and Systems

Biology, University of Edinburgh, C.H. Waddington Building, The Kings

Buildings, Edinburgh EH9 3JD, UK. ${ }^{3}$ Bioinformatics Team, University/BHF

Centre for Cardiovascular Science, University of Edinburgh, Queen's Medical

Research Institute, 47 Little France Crescent, Edinburgh EH16 4TJ, UK.
}

Published online: 01 August 2016

\section{References}

1. Yeung EN, Treskes P, Martin SF, Manning JR, Dunbar DR, Rogers SM, et al.

Fibrinogen production is enhanced in an in-vitro model of non-alcoholic

fatty liver disease: an isolated risk factor for cardiovascular events?

Lipids Health Dis. 2015;14:86. doi:10.1186/s12944-015-0069-3.

\footnotetext{
* Correspondence: N.W.Yeung@sms.ed.ac.uk

${ }^{\dagger}$ Equal contributors

'Hepatology Laboratory, Division of Health Sciences, University of Edinburgh,

Chancellor's Building, 49 Little France Crescent, Edinburgh EH16 4SB, UK
} 